\title{
Real-time observation of dynamic floor motion of the KEKB injector linac with a laser-based alignment system
}

\author{
T. Suwada, ${ }^{1,2, *}$ Y. Enomoto, ${ }^{1,2}$ K. Kakihara, ${ }^{1}$ K. Mikawa, ${ }^{1}$ and T. Higo ${ }^{1,2}$ \\ ${ }^{1}$ Accelerator Laboratory, High Energy Accelerator Research Organization (KEK), \\ 1-1 Oho, Tsukuba, Ibaraki 305-0801 Japan \\ ${ }^{2}$ School of High Energy Accelerator Science, The Graduate University for Advanced Studies (SOKENDAI), \\ 1-1 Oho, Tsukuba, Ibaraki 305-0801 Japan
}

(Received 5 September 2016; published 21 March 2017)

\begin{abstract}
A new remote-controllable sensing system for a laser-based alignment system is in development to measure slow dynamic displacements of the tunnel floor in real time at the KEKB injector linac. Although the injector linac had initially been aligned with a laser-based alignment system, we have found that, based on recent real-time observations, the transverse displacements of the tunnel floor to the laser axis caused by dynamic ground motion are not negligibly small compared with the required alignment tolerance, in terms of the straightness of the beam line. Based on spatial and temporal cross-correlation analyses performed using displacement vectors from data recorded during a nearly eight-month period, it was found that the tunnel floor moves coherently in space and time domains over the entire length of the linac on average over long periods of time, while the cross-correlation function varies irregularly and complexly over shorter periods of time. This report describes in detail the basic design, experimental results, and present status of the new remote-sensing system for real-time alignment observations at the KEKB injector linac.
\end{abstract}

DOI: 10.1103/PhysRevAccelBeams.20.033501

\section{INTRODUCTION}

In recent years, considerable research effort has been progressively directed toward longer (or larger) nextgeneration particle accelerators with higher energies and high-brightness beams in high-energy experiments. One important issue is that transverse wakefields resulting from misalignment of the components should be well controlled so as not to deteriorate beam qualities, because any deterioration in beam qualities caused by wakefield instabilities may result in unstable operation [1-4]. For this purpose, the development of techniques for high-precision alignment is clearly essential.

There has been renewed interest in the study of highprecision alignment techniques, particularly with the aim of applying them to high-energy particle accelerators. The development of alignment techniques is particularly essential in enabling high-energy linear accelerators (linacs) (1) to stabilize the acceleration and transportation of charged particle beams with high charges, and (2) to preserve beam qualities without any emittance blow-up. Addressing these two issues helps to maximize the injection rate of the beams into the storage rings.

\footnotetext{
*tsuyoshi.suwada@kek.jp.

Published by the American Physical Society under the terms of the Creative Commons Attribution 3.0 License. Further distribution of this work must maintain attribution to the author(s) and the published article's title, journal citation, and DOI.
}

The Super KEK B-Factory (SKEKB) project [5] is a next-generation B-factory under construction at KEK; it succeeded the KEK B-Factory (KEKB) project [6]. The SKEKB is an asymmetric electron-positron collider comprising 4-GeV positron (LER) and 7-GeV electron (HER) rings. The target luminosity of the SKEKB will be 40 times greater than the peak luminosity of the KEKB. The motivation of the SKEKB is the urgent need to perform high-energy flavor particle physics experiments concerning the $C P$ violation in B mesons [7]. Because the SKEKB is a factory machine, well-controlled operation and high-precision alignment for the accelerator complex are essential for maximizing injection efficiency, beam collision stability, and peak luminosity.

An optical alignment system with a high-precision telescope is widely utilized in relatively short $(<100 \mathrm{~m})$ linacs; however, alignment measurements with a resolution of $0.1 \mathrm{~mm}$ cannot be easily performed in long $(>100 \mathrm{~m})$ linacs $[8,9]$. Laser-based alignment is one of the prominent alignment techniques developed for long-distance accelerator complexes. This alignment technique is advantageous because it not only can be applied to initial alignment measurements for long linacs, but can also be utilized for regular monitoring of beam line straightness along the entire linac without interruption during operation. Based on these benefits, dynamic spatial displacements of the tunnel floor caused by ground motion can be measured in real time along the entire length of the linac.

A laser-based alignment technique using rectangular Fresnel zone plates has been developed at the Stanford 
two-mile linear accelerator [10]; this is a pioneering work in the development of laser-based alignment techniques [11] for long linacs. The alignment accuracy is determined to within approximately $100 \mu \mathrm{m}$, which is mainly due to installation inaccuracy in the positions of the physical centers of the zone plates [12]. Recently, another laserbased alignment system employing a Poisson spot [13] has been successfully put to practical use for the alignment of the European X-ray free electron laser (XFEL) facility [14]. The estimated achievable alignment accuracies range from $100 \mu \mathrm{m}$ to $200 \mu \mathrm{m}$, mainly due to distortions in the optical lenses used in the calibration procedure [15].

Our method is an alternative approach designed for the KEKB injector linac, and utilizes quadrant silicon photodiodes (QPDs). This is a conventional laser-based alignment technique in which transverse spatial displacements of the accelerator components from a long laser-based fiducial straight line can be measured with QPDs, where the laser-based fiducial is directly created as a laser axis by connecting two (or three) fiducial points.

To facilitate real-time observations of the dynamic displacements of the tunnel floor, remote-controllable QPDs were newly developed and were installed as replacements for manually controlled QPDs at multiple locations near the expansion joints of the linac building. This report describes in detail the new remote-controllable sensing system for the precise alignment of the KEKB injector linac, and discusses the consecutive observation results.

\section{KEKB INJECTOR LINAC}

\section{A. Layout of the beam line}

Here, we briefly describe the components and operation scheme for the laser-based alignment system of the injector linac. Figure 1 shows a schematic layout of the KEKB injector linac [16]. It comprises eight sectors (A-C and 1-5), which together constitute two long straight sections.
One section is $125 \mathrm{~m}$ long and is composed of sectors A and $\mathrm{B}$; the other is $476 \mathrm{~m}$ long and is composed of sectors $\mathrm{C}$ and 1-5. These two straight sections are connected to a $180^{\circ}$ arc section with a circumferential length of $31 \mathrm{~m}$.

Single-bunch electron beams can be generated from a conventional thermionic electron gun (AT gun) and are passed through a pre-injector to attain a pulse width of $\sim 10$ ps [17]. The electron gun can generate electron beams at maximum charges of $20 \mathrm{nC} /$ bunch and a maximum repetition rate of $50 \mathrm{~Hz}$ for positron production. The primary electron beams $(\sim 10 \mathrm{nC} /$ bunch $)$ can be stably accelerated to $3.3 \mathrm{GeV}$ from the end of the buncher up to a positron production target installed at the front of unit 1-5.

The positrons are captured with a flux concentrator and accelerated up to an energy of $120 \mathrm{MeV}$ using largeaperture S-band accelerating structures. The subsequently positioned normal S-band accelerating structures accelerate the positrons up to an energy of $1.1 \mathrm{GeV}$ at the second beam switchyard (SY2) and the positron beam is injected into a new damping ring (DR) [18] through the linac-to-DR transport line (LTR) [19]. The positron beams shall subsequently be transported back through the DR-to-linac transport line (RTL) to the SY2, and again accelerated up to $4 \mathrm{GeV}$ in the linac for injection into the SKEKB LER, while the electron beams can be directly accelerated up to $7 \mathrm{GeV}$ in the linac for injection into the SKEKB HER through the $180^{\circ}$ arc section where the beam energy is $1.5 \mathrm{GeV}$.

\section{B. Accelerator unit}

The 600-m-long injector linac comprises eight sectors along with a 180-degree arc section, in which the typical sector length is $76.8 \mathrm{~m}$. One sector comprises eight accelerator units, each having a length of $9.6 \mathrm{~m}$; the mechanical structure of a typical accelerator unit is described in detail elsewhere [9].

The two fiducial points are located at both ends of the accelerator unit and create a straight line parallel to the laser

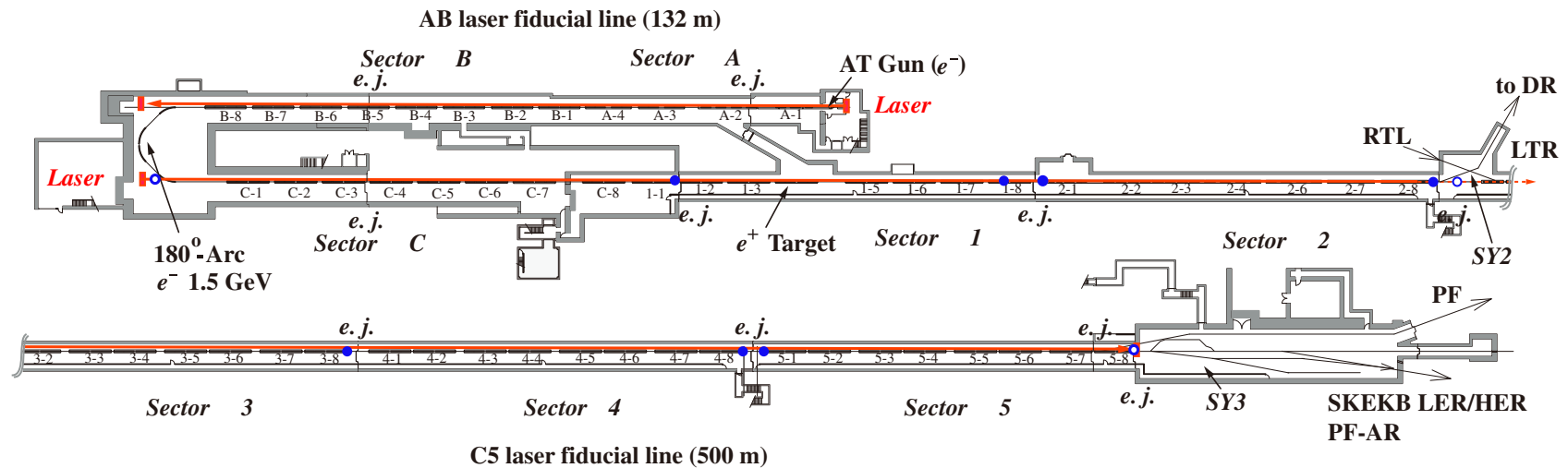

FIG. 1. Schematic layout of the KEKB injector linac and laser fiducials for two long straight sections. The abbreviation e.j. indicates an expansion joint between the building blocks of the linac. The two arrows (red) show the laser-based fiducials for two long straight sections. The solid circles (blue) represent the remote-controllable QPDs fixed to the accelerator unit and the open circles (blue) represent the reference remote-controllable QPDs fixed on the tunnel floor. 


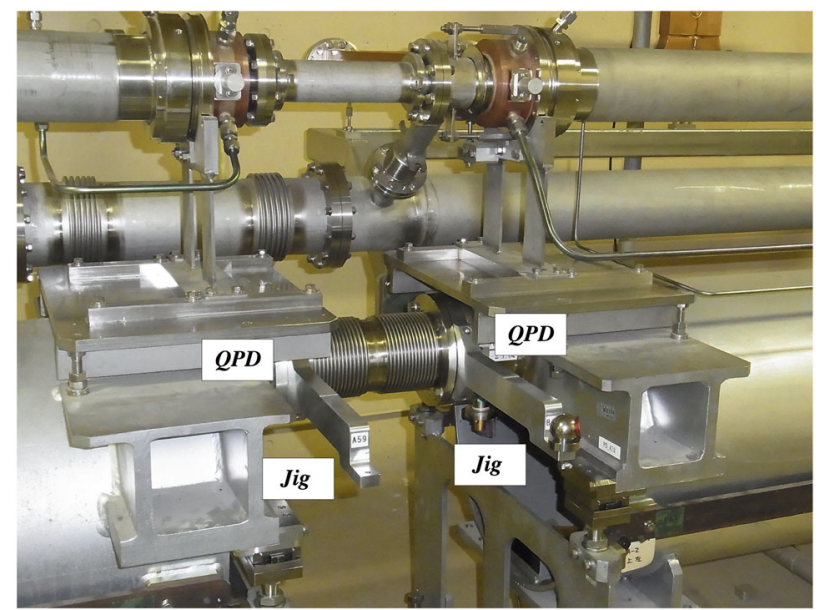

FIG. 2. Mechanical jigs for mounting the tracker target. The QPD is fixed at the end face of the accelerator unit. The jig and QPD are connected by high-precision flange-flange mechanical joining.

axis at a horizontal distance of $400 \mathrm{~mm}$, where a tracker target is mounted using a new mechanical jig, as shown in Fig. 2. The central positions of the four accelerating structures are aligned along with the central positions of the laser pipe, using a conventional alignment technique employing a laser tracker in which the two fiducial points are utilized.

Regarding the component alignment, new target bases for the accelerator structures have been developed to measure the alignment, as shown in Fig. 3. The target base is mounted on an outer surface of the rf coupler for the accelerating structure. The horizontal level of the target base is adjustable within a standard level of precision of $0.02 \mathrm{mrad}$.

Other target bases for the quadrupole magnets have also been newly fabricated and mounted. A similar alignment procedure can be applied to the quadrupole magnets. Such

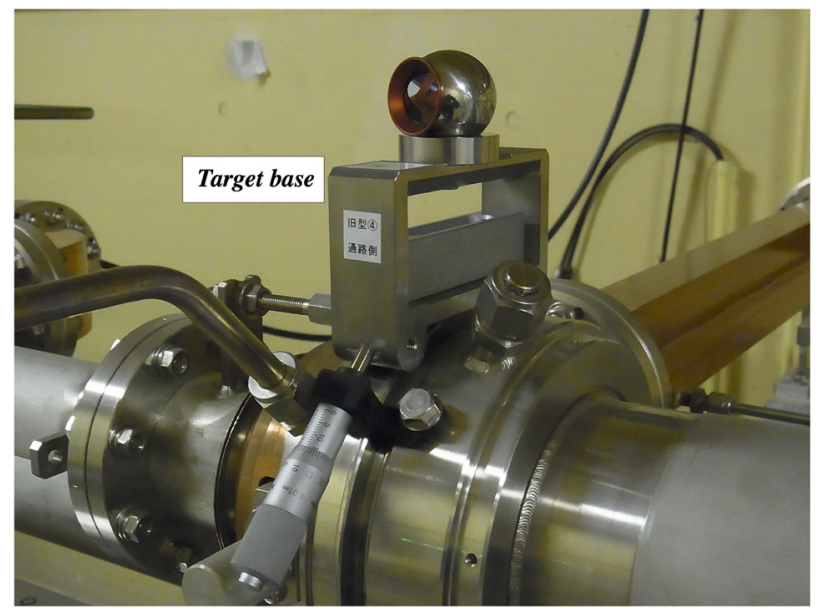

FIG. 3. Target base on the accelerating structure in the alignment measurement with a laser tracker. alignment procedures in each accelerator unit can be performed with a level of precision of $50 \mu \mathrm{m}$ in one standard deviation in both the horizontal $(x)$ and vertical (y) directions before installing the accelerator unit to the beam line.

\section{LASER-BASED ALIGNMENT SYSTEM}

\section{A. Overview}

Two independent laser-based alignment systems are available - one to align the $\mathrm{AB}$ straight section, and another to align the $\mathrm{C} 5$ straight section (see Fig. 1). The lengths of the laser-based fiducial lines for the $\mathrm{AB}$ and $\mathrm{C} 5$ straight sections are $132 \mathrm{~m}$ and $500 \mathrm{~m}$, respectively. Along with the input optical system, two laser sources with commercially available $1-\mathrm{mW}$ and $10-\mathrm{mW} \mathrm{He}-\mathrm{Ne}$ lasers are installed for the $\mathrm{AB}$ and $\mathrm{C} 5$ straight sections, respectively. Here, only the alignment system for the C5 straight section is described; however, the system for the $\mathrm{AB}$ straight section is very similar to it.

The laser source is $16 \mathrm{~m}$ upstream from the upstream end face of unit C-1. The laser passes through laser pipes in a vacuum while the laser source is installed in an atmospheric environment. In order to prevent any unnecessary reflection and scattering of the laser light, the inner surface of the laser pipe is coated with a black paint composed of acrylic resin. The laser pipes are evacuated using two scroll pumps with a pumping speed of $1000 \mathrm{l} / \mathrm{min}$; the pumps are installed $178 \mathrm{~m}$ and $321 \mathrm{~m}$ downstream from the input optical system, which is described in detail in a later section. Two vacuum windows $(20 \mathrm{~mm}$ and $15 \mathrm{~mm}$ in thickness) constructed of transparent synthetic quartz have been attached to the end faces of the initial and last laser pipes of the straight section, to maintain higher vacuum in the laser pipes. A vacuum level of a few $\mathrm{Pa}$ can be attained.

Vacuum flanges are used to precisely connect holders to both ends of the accelerator unit; a QPD with a silicon semiconductor (diameter: $10 \mathrm{~mm}$ ) is mounted at the center of the holders. The fifty accelerator units, with both regular and irregular lengths, are distributed along the C5 straight section; these units must be aligned with more than 100 manually controlled QPDs within transverse displacements of the required tolerance $(0.1 \mathrm{~mm}$ in one standard deviation) along the laser-based fiducial.

There are two fiducial points, which specify the transverse coordinates in terms of the laser axis. They are the transverse central positions of the initial and last QPDs (QPD_REF1UA and QPD_584D, respectively) installed at $z=6.3 \mathrm{~m}$ and $z=500 \mathrm{~m}$ downstream from the input optical system, respectively. These two fiducial QPDs are rigidly fixed on the floor of the accelerator tunnel after they have been locally aligned with a conventional laser tracker.

A QPD is mounted at a target point, which should be aligned in an accelerator unit; it is directly irradiated by the 
TABLE I. Summary of the expected errors and sources in the alignment measurement for an accelerator unit.

\begin{tabular}{llc}
\hline \hline Error category & Error source & Error $(\mathrm{rms})[\mu \mathrm{m}]$ \\
\hline Mechanical & QPD Mounting & 50 \\
Electrical & Detector offset & 15 \\
Laser & Profile \& Stability & 30 \\
Sum total & & 60 \\
\hline \hline
\end{tabular}

laser-based fiducial using a relatively narrow beam width. The central positions at the target can be estimated by using the QPD to measure the intensity centroid of the laser-based fiducial in the transverse directions.

Using this alignment technique, the accelerator units of the linac can be precisely aligned along the laser axis by measuring the transverse displacements from the laser fiducial, while accelerator components on the accelerator unit are aligned using the conventional laser tracker-based alignment technique described in Sec. II B.

The initial alignment of the accelerator units was carried out at the locations of the manually controlled QPDs. The expected errors and sources are summarized in Table I. The alignment accuracy for an accelerator unit is determined to be approximately $60 \mu \mathrm{m}$, and that for accelerator components is estimated within approximately $100 \mu \mathrm{m}$ in total.

\section{B. Remote-controllable QPD}

A photograph of a new remote-controllable QPD is shown in Fig. 4. The QPD is made of an iron-steel chamber (SS400) with an aperture diameter of $117 \mathrm{~mm}$, an actuator, and a QPD sensor.

A laser pipe can be directly joined to the QPD chamber via a flange connection to maintain a suitable vacuum level. The effective aperture of the QPD chamber is $92 \mathrm{~mm}$ for the relevant laser propagation.

The actuator comprises a central shaft, a compact air cylinder with a size of $16 \mathrm{~mm}$ (bore) $\times 75 \mathrm{~mm}$ (stroke) driven by a compressed-air system $\left(5 \mathrm{kgf} / \mathrm{cm}^{2}\right)$, and a micro-solenoid valve. A compact commercially available actuator was selected so it could be directly mounted on the

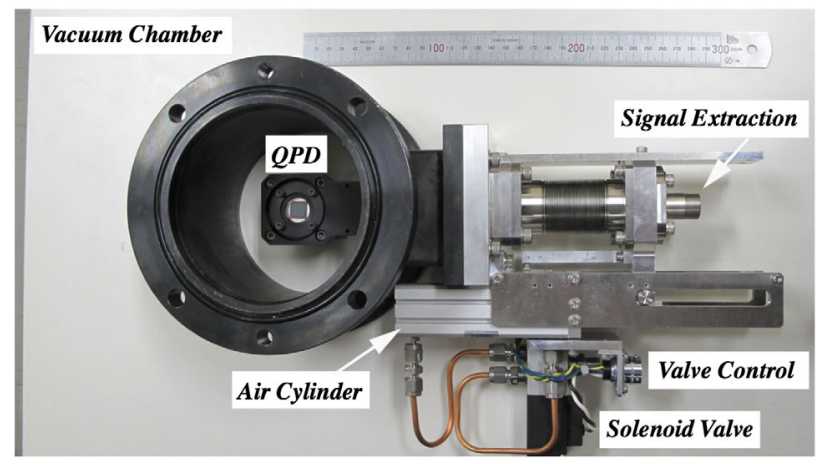

FIG. 4. Remote-controllable QPD.

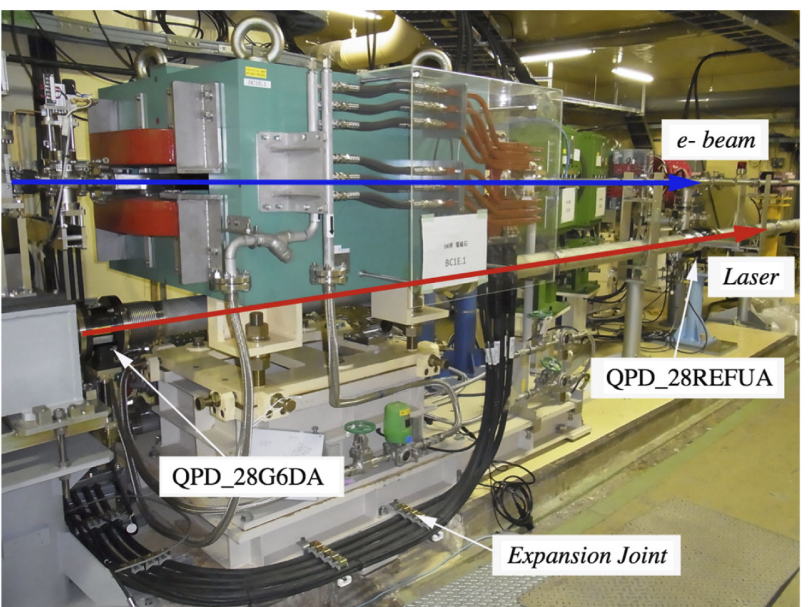

FIG. 5. Remote-controllable QPDs installed at SY2.

chamber. The driving speed of the actuator is adjustable by speed-control tuners that control the amount of compressed inlet and outlet air.

The central shaft stroke is $70 \mathrm{~mm}$, and is limited by mechanical stoppers that accurately set the central position of the QPD in terms of the laser-based fiducial line within an accuracy of $50 \mu \mathrm{m}$. The driving position of the QPD is monitored by mechanical micro switches mounted on the cylinder. The contact signals of the micro switches are sent to a local control system, which monitors the driving position of the QPD through an input/output (I/O) 1 module (TTL outputs) in a local control system.

Two remote-controllable QPDs were newly installed (or replaced manually controlled QPDs) in the middle of the injector linac during its 2014 summer shutdown, and seven remote-controllable QPDs were additionally installed during the 2015 summer shutdown at multiple locations near the expansion joints of the linac building.

Figure 5 shows a photograph of the remote-controllable QPDs installed at SY2. It can be seen that the laser-based fiducial passes through laser pipes $780 \mathrm{~mm}$ above the tunnel floor while the height of the beam line is $1200 \mathrm{~mm}$. The downstream QPD (QDP_28REFUA) is one of the fiducial points directly fixed on the tunnel floor.

\section{Local control system}

The local control system of the remote-controllable QPD is shown in Fig. 6. The local control system comprises a programmable logic controller (PLC)-based control system running on a Linux platform, and uses control software from the Experimental Physics and Industrial Control System (EPICS) environment [20]. It is installed at a monitor control station in the klystron gallery; in addition, a local controller is installed in the linac tunnel. The local control system is connected to the Linux-based main control system through an Ethernet-based linac control network. 
Linac control network

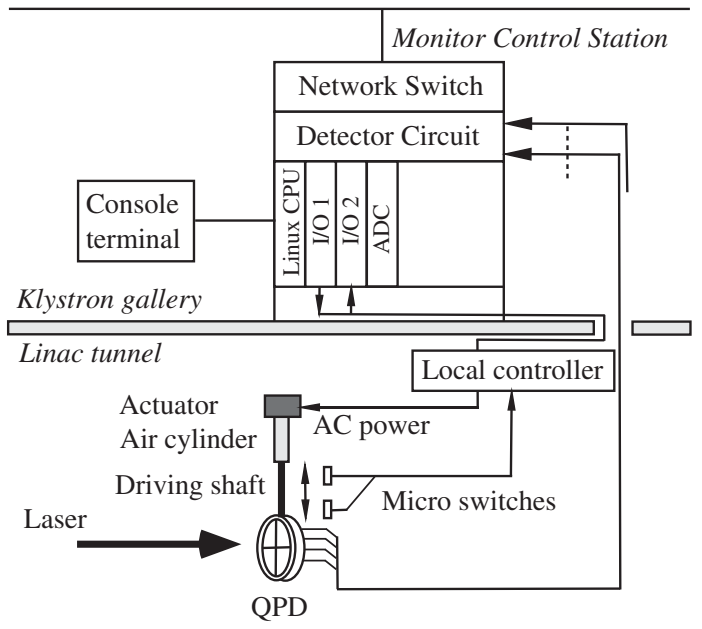

FIG. 6. Schematic block diagram of the local control system.

The system controls the "Open/Close" of a solenoid valve (the load current is $48 \mathrm{~mA}$ ) through an I/O 2 module (24-VDC inputs) in the local control system by switching a 200-VAC power supply on and off with a relay switch, which can be remotely controlled in the local controller; it is also used to test the QPD drive and to tune the speed of the actuator via a manual switch.

\section{Laser source and input optical system}

The laser source and optical lens configuration in the input optical system are shown in Fig. 7. The input optical system is mounted on a standard $1500 \times 900 \mathrm{~mm}^{2}$ optical table in an atmospheric environment. The entire input optical system is covered with an acrylic box; furthermore, it is fully enclosed in a plastic sheet to minimize the influences and variations of the atmospheric environment. The optical table is mounted on a rigid iron girder fixed to a 20 -mm-thick iron plate. This plate is fixed to an isolated floor with an area of $500 \times 1510 \mathrm{~mm}^{2}$. This isolated floor is structurally separated from the tunnel floor by means of a $100 \mathrm{~mm}$ construction gap.

The input optical system is based on a dioptric system using dioptric lenses and reflecting mirrors. The transverse width of the laser beam is $W_{x}\left(\right.$ or $\left.W_{y}\right) \simeq 0.83 \mathrm{~mm}$ at a point $10 \mathrm{~cm}$ downstream from the front end face of the laser tube, and its divergence is $\sim 0.7 \mathrm{mrad}$. Here, it should be mentioned that the beam width is defined by the foursigma width of an intensity distribution projected onto the $x$ or $y$ axis of the two-dimensional intensity profile. The beam width is slowly expanded to $W_{x}\left(\right.$ or $\left.W_{y}\right) \simeq 29 \mathrm{~mm}$ at the outlet of the input optical system with five spherical planoconvex lenses (BK7, focal length: $f=50,150,300,1500$, and $5000 \mathrm{~mm}$ ) mounted on the optical table. The total path length of the laser beam is $\sim 5.5 \mathrm{~m}$ in the optical table. This relatively long path length has been carefully designed to

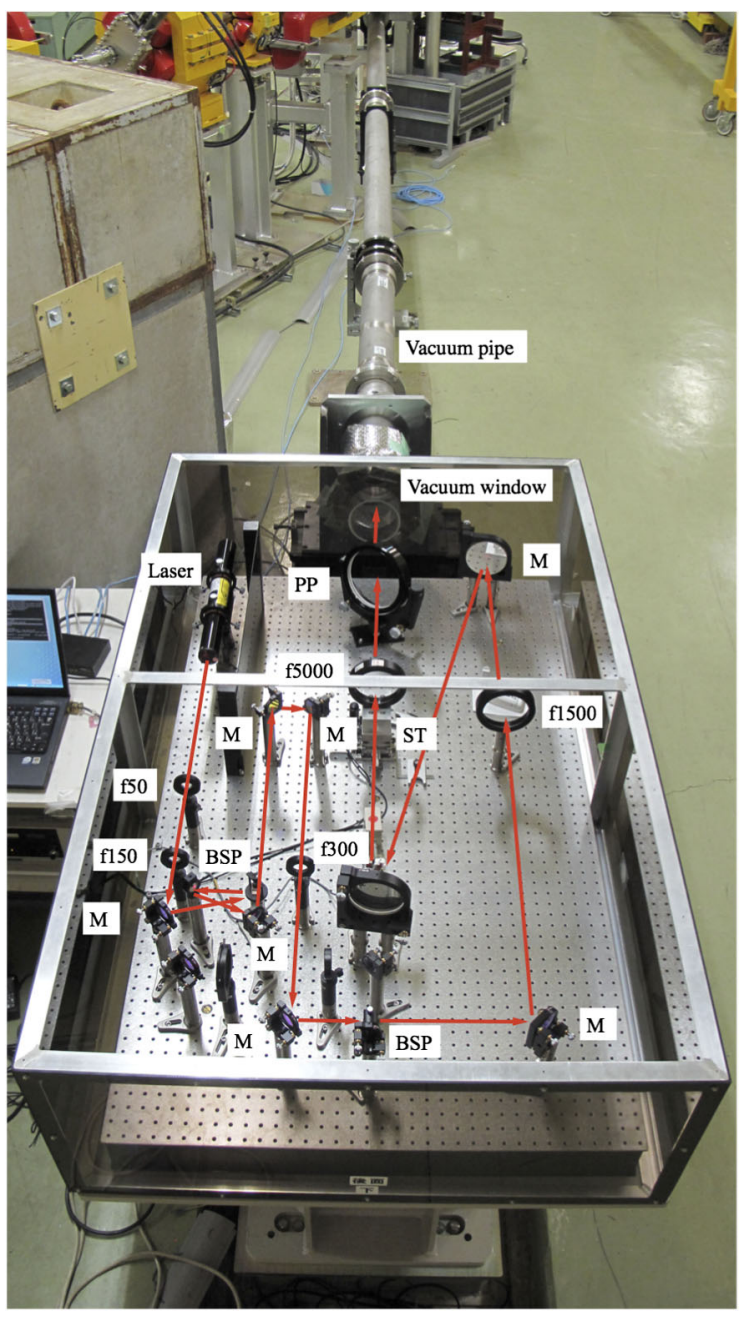

FIG. 7. Input optical system with a 10-mW He-Ne laser for the C5 straight section. The laser beam is delivered from the input optical system to the end point of the linac through metallic vacuum pipes (to top direction). M: reflecting mirror, BSP: beam splitter, ST: linear stage, PP: parallel plate, f: focusing lens. It can be seen that the floor for the input optical system is structurally separated from the tunnel floor.

create a beam profile that can pass through the narrow vacuum pipes.

The propagation direction of the laser beam should be adjusted in order to be as precisely straight as possible (with respect to the two fixed QPDs) without any unnecessary diffraction and instability. The injection laser beam to the vacuum pipes must allow the injection angles and transverse positions to be adjusted in both the $x$ and $y$ directions at the input optical system. The injection angles are adjusted by moving the final spherical plano-convex lens ( $f 5000)$ in the perpendicular plane with respect to the beam axis. This lens is mounted on high-precision translation stages with pico-motor actuators, which can control the injection angles with minimum horizontal and vertical angle steps of $1.8 \mathrm{nrad}$ and $0.73 \mathrm{nrad}$, respectively, via a stage controller. 
The injection angles can be automatically adjusted with a feedback control. On the other hand, the transverse positions are manually adjusted by tilting a parallel plate located after the final lens. This parallel plate has an antireflective (AR) coating on both its surfaces; its thickness and diameter dimensions are $20 \mathrm{~mm}$ and $150 \mathrm{~mm}$, respectively. Thus, the transverse positions of the laser beam can be adjusted finely without changing any injection angles. The propagation and stability characteristics of the laser-based fiducial are described in detail elsewhere [21].

\section{E. Detector circuit}

A block diagram of the detector circuit in the local control system is shown in Fig. 8.

The detector circuit comprises four independent current integrators that convert the photocurrents $\left(I_{1}-I_{4}\right)$ to the corresponding voltages $\left(V_{1}-V_{4}\right)$ with the use of a highprecision feedback resistor of $33.33 \mathrm{k} \Omega$ with a precision of $\pm 0.02 \%$, which is the required precision for the detector circuit.

The typical photocurrent from a QPD is spread over a range of $70 \mu \mathrm{A}$ to $150 \mu \mathrm{A}$ depending on the location, and the voltage signals are fed into a 16-bit analog-to-digital converter (ADC) for differential voltage input through buffer amplifiers. They are also output to monitor outputs $\left(V_{m 1}-V_{m 4}\right)$ through other buffer amplifiers for monitoring the signal intensities from the detector circuit.

Because our QPD measurement system is basically destructive, it is not possible to perform the displacement measurements for all the QPDs at one time; thus, the QPD signals should be remotely selected with relay switches

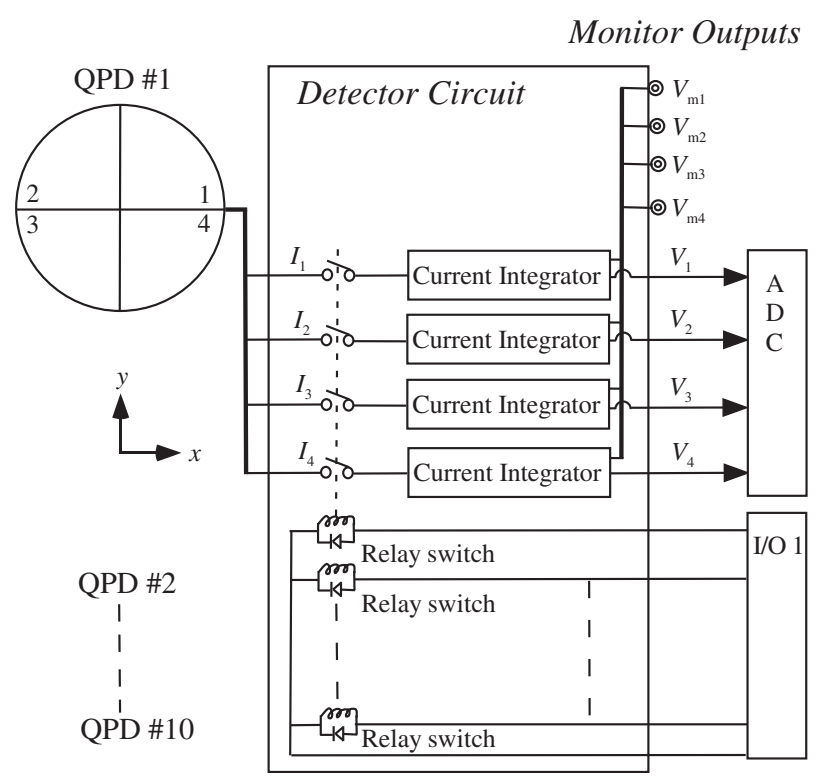

FIG. 8. Schematic block diagram of the detector circuit in the local control system. through a digital input/output module (I/O 1, TTL outputs) in the local control system (see Fig. 6).

The transverse displacements $(x, y)$ of the accelerator unit in terms of the laser axis are derived on the basis of the QPD measurement by

$$
\begin{gathered}
V_{H}=\left(V_{2}+V_{3}\right)-\left(V_{1}+V_{4}\right), \\
V_{V}=\left(V_{3}+V_{4}\right)-\left(V_{1}+V_{2}\right), \\
V_{S}=\sum_{k=1}^{4} V_{k}, \\
x=S_{x} \cdot\left(V_{H} / V_{S}\right), \\
y=S_{y} \cdot\left(V_{V} / V_{S}\right) .
\end{gathered}
$$

Here, $V_{k}(k=1-4)$ is an output voltage of the $k$ th channel of the QPD. $S_{x}$ and $S_{y}$ are the sensitivity coefficients for the $x$ and $y$ displacements, respectively.

\section{F. Data acquisition procedure}

Server programs that facilitate QPD measurements are always running in the main control system. Every four hours on the hour, the basic functions provided by the server are executed in order to record a series of displacement data for all the remote-controllable QPDs. The basic functions comprise (1) feedback control for the laser axis, (2) actuator control for the QPD, and (3) recording displacement data for the QPD.

When the server sends a control request to the local control system every four hours on the hour, the local control system receives the control request and a series of tasks in the basic functions are executed. First, the end positions of the laser axis at the last QPD are stabilized by the feedback control until the transverse positions of the laser axis are within the allowable region of $\pm 50 \mu \mathrm{m}$ from the center of the last QPD. Second, the most upstream QPD is driven to the central position of the laser pipe. Third, displacement data from the QPD are repeatedly obtained one thousand times; from this data, the average and standard deviation values are calculated in the four output voltages along with the $x$ and $y$ displacements. Fourth, the most upstream QPD is driven from the central position to the home position.

Thus, a series of basic functions similarly work toward the next QPD one-by-one in order, in the downstream direction. For all the remote-controllable QPDs, the series of basic functions is repeated nine times in total; it takes approximately five minutes to execute.

\section{G. Feedback control of the laser axis}

The laser-based fiducial is directly created as a laser axis by connecting two fiducial points, to which the centers of 
the initial and last QPDs are assigned. The transverse positions of the laser axis at the initial QPD are manually adjusted by using the parallel plate to translate the laser beam at the input optical system, in order to correspond to the center of the initial QPD. Meanwhile, the pointing of the laser beam is stabilized at the last QPD by controlling the $x$ and $y$ injection angles at the input optical system, based on a feedback control.

The feedback control system for stabilizing the laser axis at the last QPD is shown in Fig. 9.

Four photocurrents from the last QPD are converted to four corresponding voltages in a detector circuit. The detector outputs the four voltages proportional to the intensities of the photocurrents. The voltage signals are directly fed into an ADC module in the local control system, where both the $x$ and $y$ positions are calculated using Eqs. (1)-(5).

The averaged transverse position data are sent to the main control system every $\sim 1 \mathrm{~s}$ via an Ethernet-based local area network (LAN). The translational linear stage, on which the final plano-convex lens is mounted, controls its $x$ and $y$ positions via the feedback control. Thus, the $x$ and $y$ injection angles are automatically controlled and the transverse positions of the laser axis are stabilized around the center of the last QPD every four hours on the hour just before the start of the data acquisition procedure.

The pointing stability of the laser beam in the $x$ and $y$ axes is $25 \mu \mathrm{m}$ in one standard deviation. The results obtained in the $x$ direction are shown in Fig. 10. The obtained pointing stabilities are sufficiently met with the high-precision laser-based alignment measurement technique required for the injector linac (see [21] for a detailed description).

\section{H. Calibration procedure}

While the transverse displacements of the QPD center from the laser axis can be basically derived on the basis of calculations in Eqs. (1)-(5), the sensitivity coefficients should be measured by following a calibration procedure. The laser axis is stabilized at the center of the last QPD with the feedback control on, and the feedback control can be similarly applied to the laser axis in the calibration procedure.

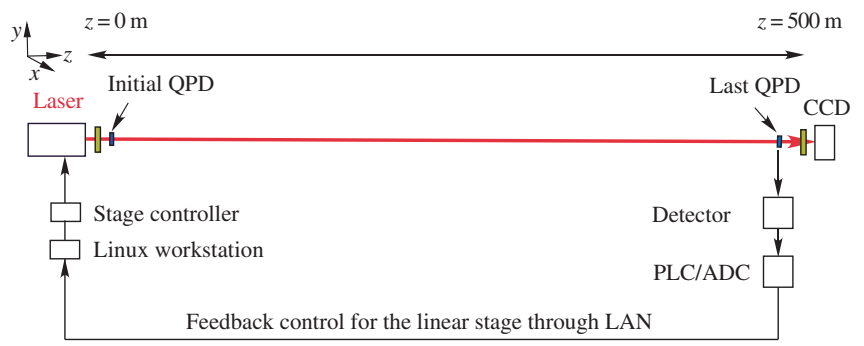

FIG. 9. Feedback control system for stabilizing the laser axis at the last QPD.

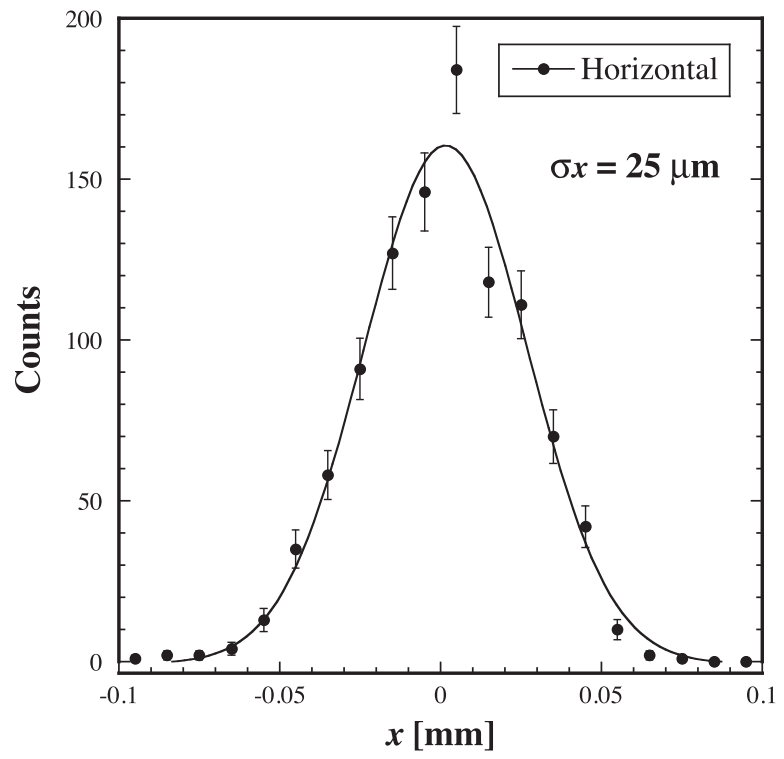

FIG. 10. Distribution plots for the pointing stabilities of the laser beam in the $x$ axis.

The transverse positions of the laser axis at the last QPD are controlled with the feedback control by changing the target position in 1-mm steps; these changes in the target position automatically control the injection angles of the laser axis in the input optical system. Because the displacement of the laser axis at each QPD is proportional to the distance from the laser source, the sensitivity coefficients can be easily derived on the basis of the linear relation of the displacement to the distance while assuming the straightness of the laser axis.

Figure 11 shows the result obtained in the calibration procedure for all the remote-controllable and reference QPDs in the horizontal direction. A similar result was obtained for the calibration procedure in the vertical direction.

A commercially available beam profiler was used to measure a two-dimensional intensity image of a cross section of the laser axis; the image was obtained through a vacuum window installed after the last QPD. The twodimensional intensity image can be obtained by a beam profiler with an aperture of $36 \times 24 \mathrm{~mm}^{2}$ and an effective pixel count of $4008 \times 2672$. The transverse positions of the laser axis were measured by fitting a two-dimensional Gaussian function to the intensity distribution. The position calibration for the laser axis at the last QPD was successfully performed on the basis of this procedure.

In the calibration procedure performed prior to the installation of the remote-controllable QPDs, the calibration had been performed by moving manually controlled QPDs installed in every sector in both horizontal and vertical directions, with a step length of $0.5 \mathrm{~mm}$ over ranges of $\pm 3 \mathrm{~mm}$, while keeping the laser beam fixed [9]. The new calibration procedure successfully reduces the time 


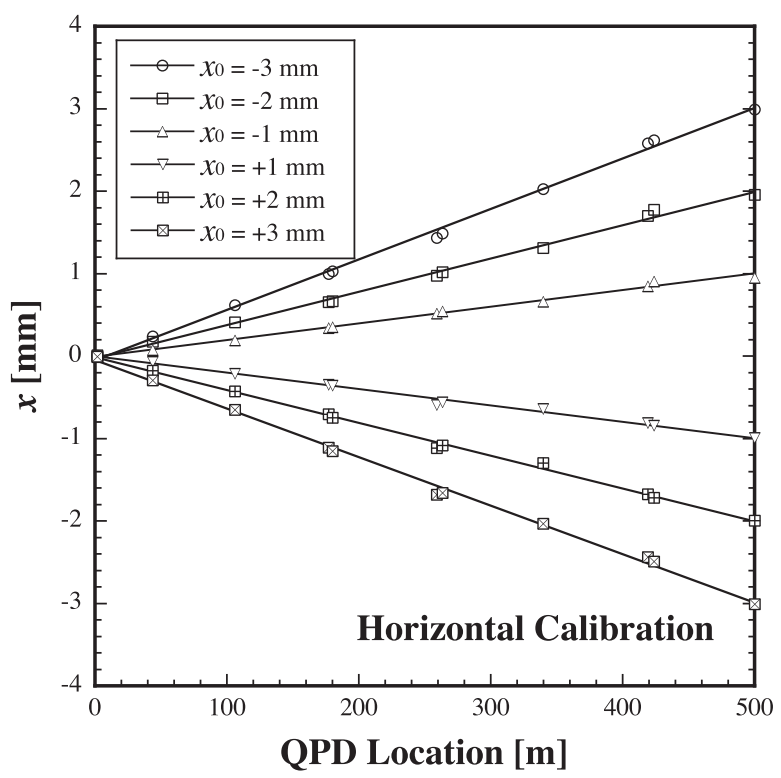

FIG. 11. Results from the horizontal calibration procedure for all the remote-controllable and reference QPDs. It should be noted that a laser source and the fiducial QPD are located at $z=0$ and $z=500 \mathrm{~m}$, respectively. The target positions controlled with the feedback control are defined by $x_{0}$ at $z=500 \mathrm{~m}$.

consumed (approximately twelve minutes per QPD) in comparison with the previous calibration procedure. However, it should be noted that it is principally difficult to obtain the sensitivity coefficients for the initial QPD_REF1UA in the new calibration procedure because the length between the laser source and the initial QPD is too short to be obtained accurately. Thus, a series of

TABLE II. Summary of the locations of the remote-controllable QPDs and expansion joints (e.j.) along the injector linac from the laser source, and $S_{x}$ and $S_{y}$ in the $x$ and $y$ directions, respectively, in the corresponding QPD sensitivity coefficients.

\begin{tabular}{llccc}
\hline \hline QPD & e.j. & Location $[\mathrm{m}]$ & $S_{x}[\mathrm{~mm}]$ & $S_{y}[\mathrm{~mm}]$ \\
\hline REF1UA & & 1.74 & 11 & 20 \\
& C3D & 44.31 & $\ldots$ & $\ldots$ \\
11DA & & 106.11 & 12.4 & 14.3 \\
& 11D & 106.72 & $\ldots$ & $\ldots$ \\
1814DA & & 177.04 & 8.06 & 8.41 \\
& \multirow{2}{*}{ 1814D } & 178.39 & $\ldots$ & $\ldots$ \\
21UA & & 180.17 & 8.10 & 8.59 \\
28G6DA & & 259.07 & 7.15 & 7.29 \\
& \multirow{2}{*}{ 28G6D } & 259.64 & $\ldots$ & $\ldots$ \\
28REFUA & & 263.32 & 6.96 & 7.14 \\
38DA & & 339.58 & 6.68 & 5.86 \\
& \multirow{2}{*}{ 38G5U } & 341.60 & $\ldots$ & $\ldots$ \\
48DA & & 419.08 & 6.37 & 5.54 \\
51UA & \multirow{2}{*}{ 48G5U } & 421.11 & $\ldots$ & $\ldots$ \\
& & 423.65 & 6.30 & 5.40 \\
584D & 57G7U & 498.01 & $\ldots$ & $\ldots$ \\
\hline \hline
\end{tabular}

calibration procedures for other remote-controllable QPDs were stably performed in both the $x$ and $y$ directions (except for the initial QPD) without any difficulties.

The obtained sensitivity coefficients are summarized in Table II, along with the locations of the remote-controllable QPDs (except for the last fiducial QPD, QPD_584D), and the locations for expansion joints along the injector linac from the laser source. Here, it should be noted that the remotecontrollable QPDs, REF1UA and 28REFUA, were installed directly on the floor, while other manual-controllable QPDs were replaced with new remote-controllable QPDs.

\section{REAL-TIME OBSERVATION ON DYNAMIC FLOOR MOTION}

\section{A. Long-term variations of the displacement vectors in time traces}

Typically, the $x$ and $y$ ingredients of the displacements of accelerator components in rectangular coordinates are independently analyzed using time series data. However, in these analyses, it is important to take the displacement vector directly into account instead of using the $x$ (or $y$ ) ingredient, because no special directivity may be provided in the direction of the displacement vector, owing to ground motion. Moreover, it is advantageous to apply the displacement vector to the analysis with strong $x-y$ coupling, which is expected in and around the expansion joints.

Figure 12 shows the time traces of the norm and phase in the displacement vectors for the accelerator units (or tunnel floor) at multiple locations, as measured by the remotecontrollable QPDs during an approximately eight-month period starting on 7 January 2016. The norm $(r)$ and phase $(\theta)$ of the displacement vector are defined in the perpendicular plane with respect to the laser axis by

$$
r=\sqrt{x^{2}+y^{2}}, \quad \tan \theta=y / x .
$$

During the term, the end positions of the laser axis were sufficiently stabilized to the center of the last QPD with the feedback control on. Here, it should be noted that the data in the displacement vectors represent the amount of variation, that is, the differentials from the corresponding initial data.

It can be understood that the norms of the displacement vectors monotonically increase (except for that of QPD_REF1UA, which is nearest to the laser source), while it can be seen that there are some incomprehensible fine structures in each time trace. The variations in the maximum norm $\left(r_{m}\right)$ of the displacement vector in each time trace as a function of the QPD location are shown in Fig. 13(a). The maximum norms are spread over a range of $0.19-0.88 \mathrm{~mm}$ depending on the QPD location, and the average maximum norm $\left(\left\langle r_{m}\right\rangle\right)$ is $0.65 \mathrm{~mm}$ (except for that of QPD_REF1UA). 

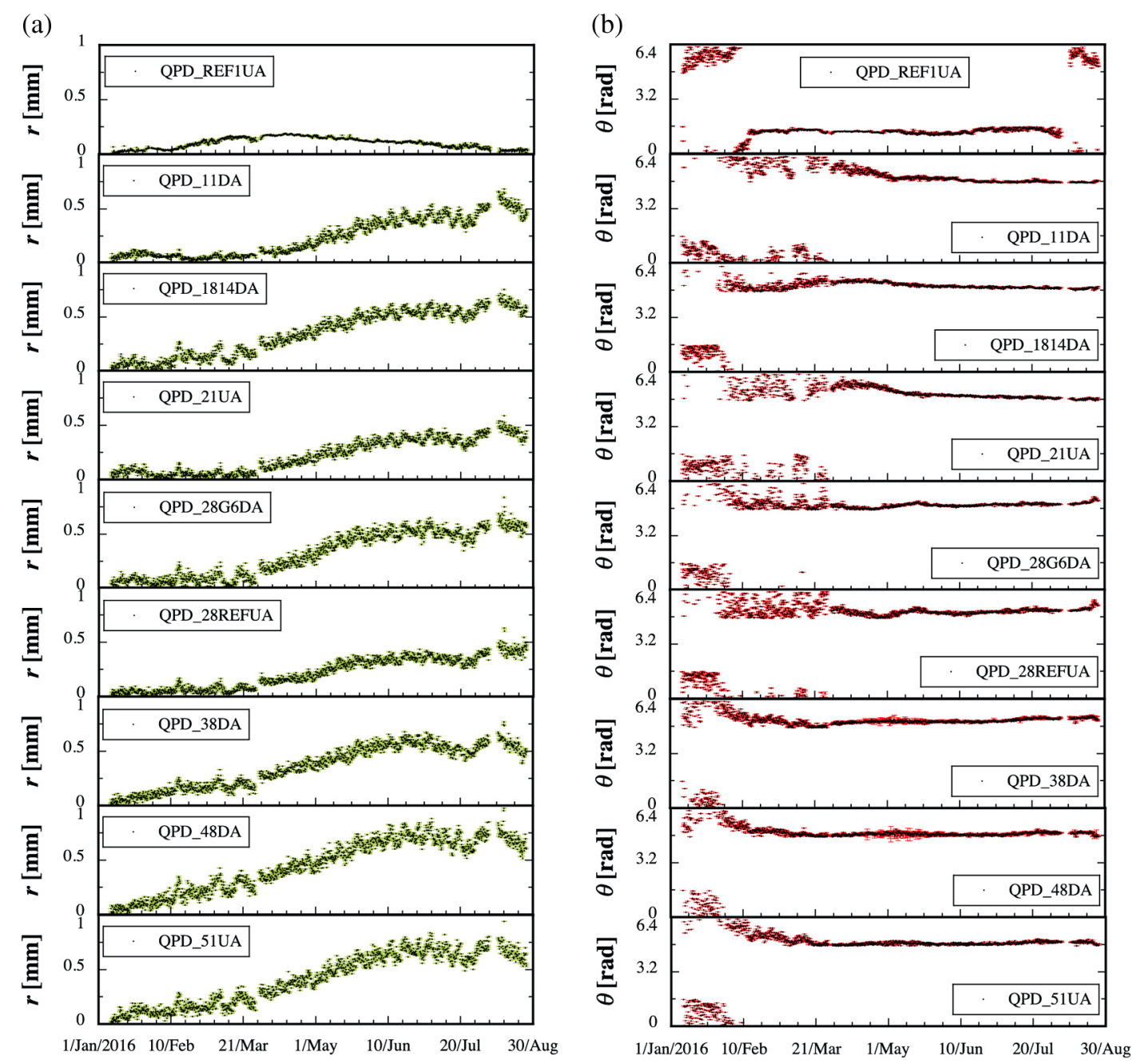

FIG. 12. Time traces in the (a) norm and (b) phase of the displacement vector for the accelerator units (or tunnel floor) measured by the remote-controllable QPDs.

The variations in the maximum growth rates $\left(s_{r}\right)$ in the time traces as a function of the QPD location are shown in Fig. 13(b). The maximum growth rates of the norms are spread over a range of 3.3-6.6 $\mu \mathrm{m} /$ day depending on the QPD location, and the average maximum growth rate $\left(\left\langle s_{r}\right\rangle\right)$ is $4.9 \mu \mathrm{m} /$ day (except for that of QPD_REF1UA).

It can also be found that the variations in the phase of the displacement vector in each time trace become almost constant starting in the beginning of May 2016, while it can be seen that there are some steep variations depending on the QPD locations. Here, it should be noted that stepwise phase variations in the time traces normally arise over $2 \pi$ in phase region 0 because the phase is defined by an angular range of $0-2 \pi$. However, it can be clearly seen that a relatively large stepwise jump arises on 28 Jan 2016 in the time traces of the phases for QPD_1814DA and QPD_28REFUA. The jump occurs over approximately four days; on the other hand, the variations in the norms of the displacement vector during the corresponding term are less than $0.2 \mathrm{~mm}$. It can be understood that during the corresponding term, the displacement vector of the accelerator unit has rotated rather than increased in the transverse plane. By using such a vector representation in the polar coordinates, for the first time, complex and dynamic behavior on the tunnel floor can be seen much more clearly compared to the previous analysis.

There are numerous factors that cause dynamic displacements of the tunnel floor, including ambient temperature, sunlight, atmospheric pressure, displacement of underground water, oceanic tides and waves, and diffusion of the ground surface. Several factors may directly account for dynamic ground motion, and other factors may secondarily cause it.

The 500-m-long linac building has two floors, which together constitute the klystron gallery and the in-ground tunnel. The tunnel floor is located 5.65 m below the Earth's surface, where the klystron gallery is situated. The temperature in the tunnel environment is stabilized with air conditioners within $25 \pm 1{ }^{\circ} \mathrm{C}$.

The linac building comprises eight building blocks that are joined to be linearly aligned with seven expansion 

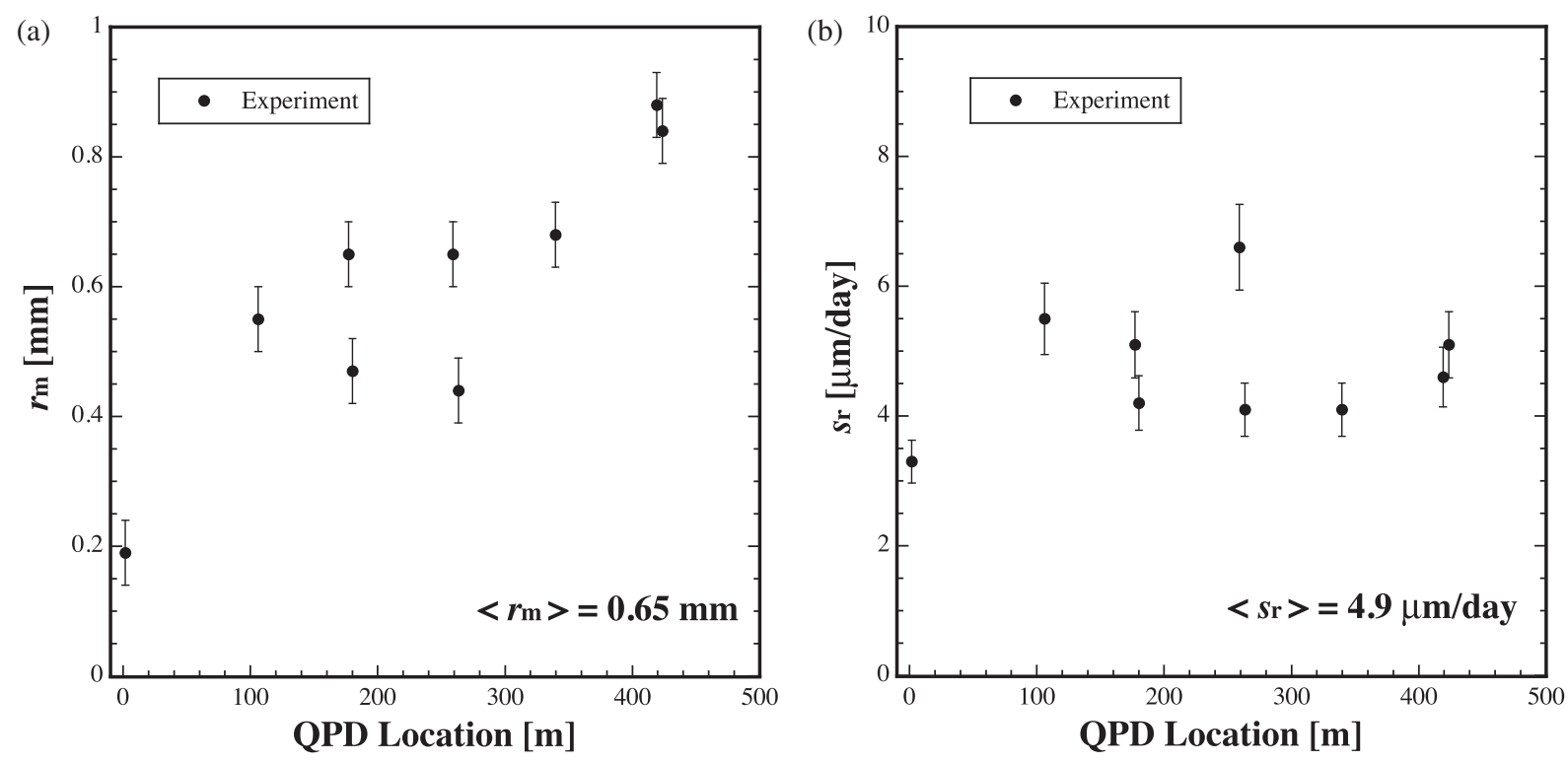

FIG. 13. Variations of the (a) maximum norm and (b) maximum growth rate in the displacement vector measurements as a function of the QPD location.

joints, which can absorb a certain amount of elastic deformation caused by expansion or contraction of the building blocks. However, residual amounts of elastic deformation may enhance deformation of the building blocks themselves. This may increase the displacement of the accelerator units through displacements of the tunnel floor, triggered by the complex processes that were mentioned previously, because the tunnel floor level may not be very deep under the ground.

In particular, it should be noted that the fiducial points created by the laser axis can move dynamically and independently in proportion to the displacement vector of the tunnel floor, even though the last fiducial point is strictly fixed at the last QPD center with the feedback control on.

Here, it is useful to discuss the differences in the dynamic floor motion of the KEKB injector linac and the ring sites, using data obtained over long periods of time. KEKB is an energy asymmetric $e^{+} e^{-}$collider with electron and positron energies of $8 \mathrm{GeV}$ (HER) and $3.5 \mathrm{GeV}$ (LER), respectively. The full operation of the physics experiment started in June 1999, and was discontinued in June 2010.

The circumference of the KEKB rings is approximately $3016 \mathrm{~m}$. The arc section of the tunnel and the basement floors of the experimental building were located $12 \mathrm{~m}$ and $16 \mathrm{~m}$ below the ground level, respectively. Thus, KEKB was located at a position approximately $6.4 \mathrm{~m}$ deeper than that of the injector linac.

During the operation of KEKB, the circumference deviation from a reference orbit was monitored by measuring closed orbit distortion (COD) with a continuous orbital feedback system. The measurement and COD correction methods are described in detail elsewhere [22]. The circumference deviation of the LER was approximately $2.5 \mathrm{~mm}$ at the maximum during a four-month period from 1 March to 30 June 2002 [22]. Assuming that the circumference deviation simply leads to the enlargement (or reduction) of the ring diameter on average, the horizontal displacement of the KEKB tunnel floor is estimated to be approximately $0.4 \mathrm{~mm}$ on average. Furthermore, by analyzing variances in the circumference, the maximum growth rate of the KEKB tunnel floor is estimated to be approximately $5 \mu \mathrm{m} /$ day on average [23]. On the other hand, at the injector linac site, the norm in the displacement vector measurements is approximately $0.65 \mathrm{~mm}$ on average along the entire linac length.

It can be understood that, on the whole, the displacement of the tunnel floor at the injector linac site is greater than that at the ring site. This could be speculated by considering the differences in the tunnel depth and building structure, while the growth rates of both tunnel floors are in good agreement with each other on average. A detailed understanding of the obtained results, which may be dependent on numerous environmental factors, could be obtained in possible future measurements.

\section{B. Cross-correlation analysis on the displacement vectors}

It is generally valid to apply cross-correlation analyses in order to capture features in their mutual relations between different multivariate vector variables in space and time domains [24]. By performing cross-correlation analyses of the time traces of the displacement vectors, variations in a cross-correlation function can be obtained in real time. This is a powerful method that provides, for the entire length of the linac tunnel, an effective understanding of localized accelerator unit displacements as well as structural building block deformation caused by dynamic ground motion. 

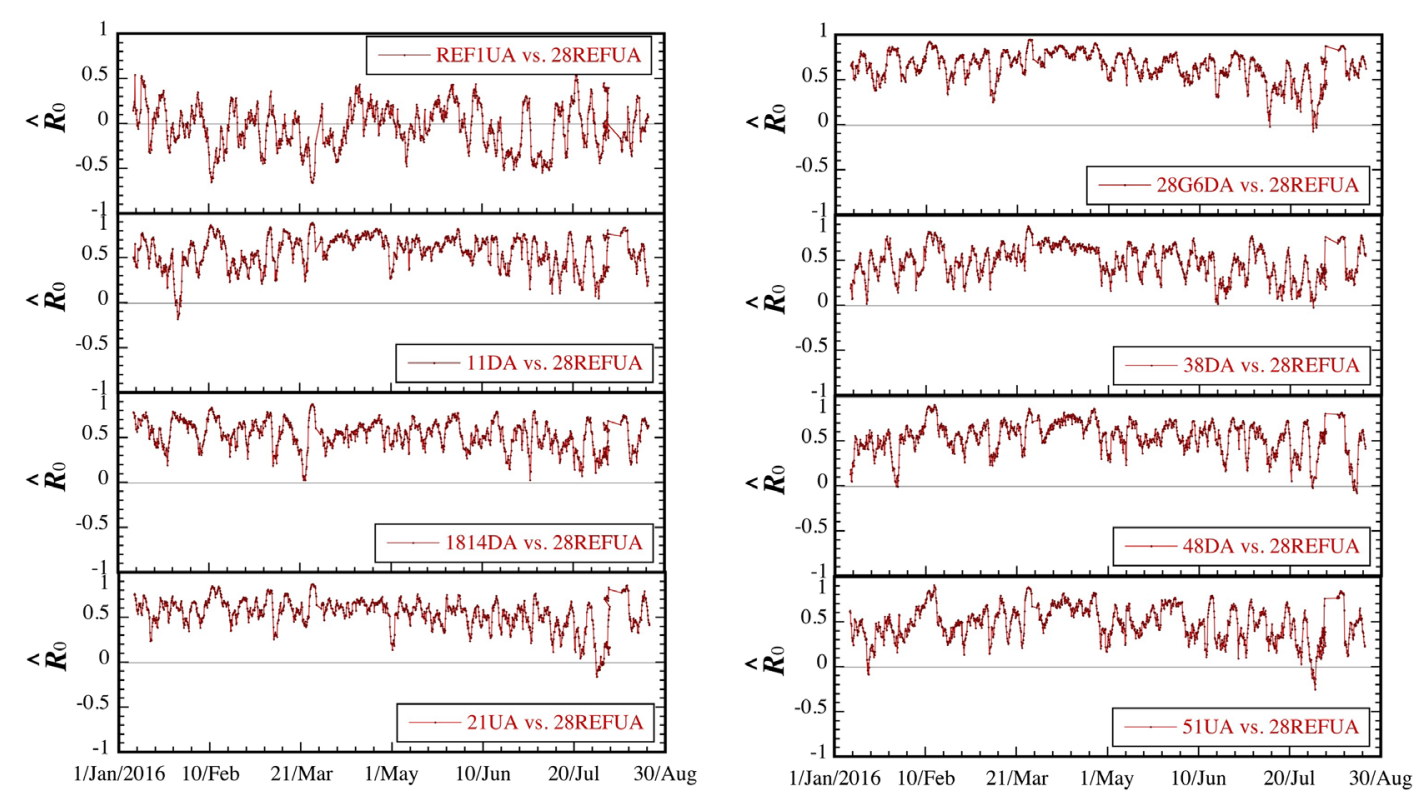

FIG. 14. Time traces in the spatial cross-correlation function of the displacement vectors, for comparisons between the reference QPD (QPD_REF28DA) and other remote-controllable QPDs for the accelerator units during the same term.

A cross-correlation function $\left(\hat{R}_{k}(i, j)\right)$ between the $n$th displacement vectors $\left(\vec{d}_{n}(i)\right.$ and $\left.\vec{d}_{n}(j)\right)$ obtained for the $i$ th and $j$ th QPDs, respectively, in a time series with a time lag of $k$ is defined by

$$
\begin{gathered}
\langle\vec{\mu}(i)\rangle=\frac{1}{N} \sum_{n=1}^{N} \vec{d}_{n}(i), \\
\hat{C}_{k}(i, j)=\frac{1}{N} \sum_{n=k+1}^{N}\left[\vec{d}_{n}(i)-\langle\vec{\mu}(i)\rangle\right] \cdot\left[\vec{d}_{n-k}(j)-\langle\vec{\mu}(j)\rangle\right], \\
\hat{R}_{k}(i, j)=\frac{\hat{C}_{k}(i, j)}{\sqrt{\hat{C}_{0}(i, i) \hat{C}_{0}(j, j)}} .
\end{gathered}
$$

Here, a dot in Eq. (8) indicates a scalar product for the displacement vectors, and $\langle\vec{\mu}(i)\rangle$ is an average of the $i$ th displacement vector in a time series with a data length of $N$. $\hat{C}_{k}(i, j)$ is a cross-covariance function, which is defined similarly to the cross-correlation function. It should be noted that $\hat{R}_{k}(i, j)$ is normalized by using cross-covariance functions $\hat{C}_{0}(i, i)$ and $\hat{C}_{0}(j, j)$ for the $i$ th and $j$ th QPDs, respectively, without any time lag $(k=0)$. Thus, $\hat{R}_{k}(i, j)=$ $1(-1)$ shows strong positive (negative) correlation, while $\hat{R}_{k}(i, j)=0$ shows no correlation, and $\hat{R}_{k}(i, j)$ at $k=0$ $(k>0)$ stands for the cross-correlation function at the present (or a past) time in a time series.

Figure 14 shows the time traces in the cross-correlation function $(k=0)$ of the displacement vectors between the reference QPD (QPD_REF28DA) and other remotecontrollable QPDs for the accelerator units during the same term. This correlation is related to that between two displacement vectors in the space domain (spatial cross-correlation). Here, QPD_REF28DA is installed near the middle of the injector linac.

It can be seen that each cross-correlation function varies irregularly in the corresponding time trace; however, the averages in the time traces decrease in the range of $\hat{R}_{0}=$ $0.65 \pm 0.01$ to $0.48 \pm 0.01$ in accordance with the distance from the reference QPD (except for QPD_REF1UA, where $\hat{R}_{0}=-0.04 \pm 0.01$ ). The average of $\hat{R}_{0}$ is $0.54 \pm 0.01$, and the results are shown in Fig. 15(a).

This means that the relatively strong positive crosscorrelations show relatively high coherency in the dynamic displacements of the accelerator units along the entire length of the linac tunnel, except for QPD_REF1UA. In other words, the results show that the floor levels in each building block move coherently with each other on average in the time series.

It should be noted that the time trace in the crosscorrelation function for QPD_REF1UA is markedly different from those in the other cross-correlation functions. This may have occurred because the floor for the laser source is structurally separated from the tunnel floor of the building block (see Fig. 7). Thus, the dynamic displacements of the laser source can be mainly dominated by ground motion, because the laser source cannot be strongly affected by deformation of the linac building. It seems that QPD_REF1UA stands alone from the other building blocks.

Figure 16 shows the time traces in the cross-correlation function $(k>0)$ of the displacement vectors between the reference QPD and other remote-controllable QPDs for the accelerator units during the same term. This correlation is related to that between two displacement vectors in the time domain (temporal cross-correlation). 

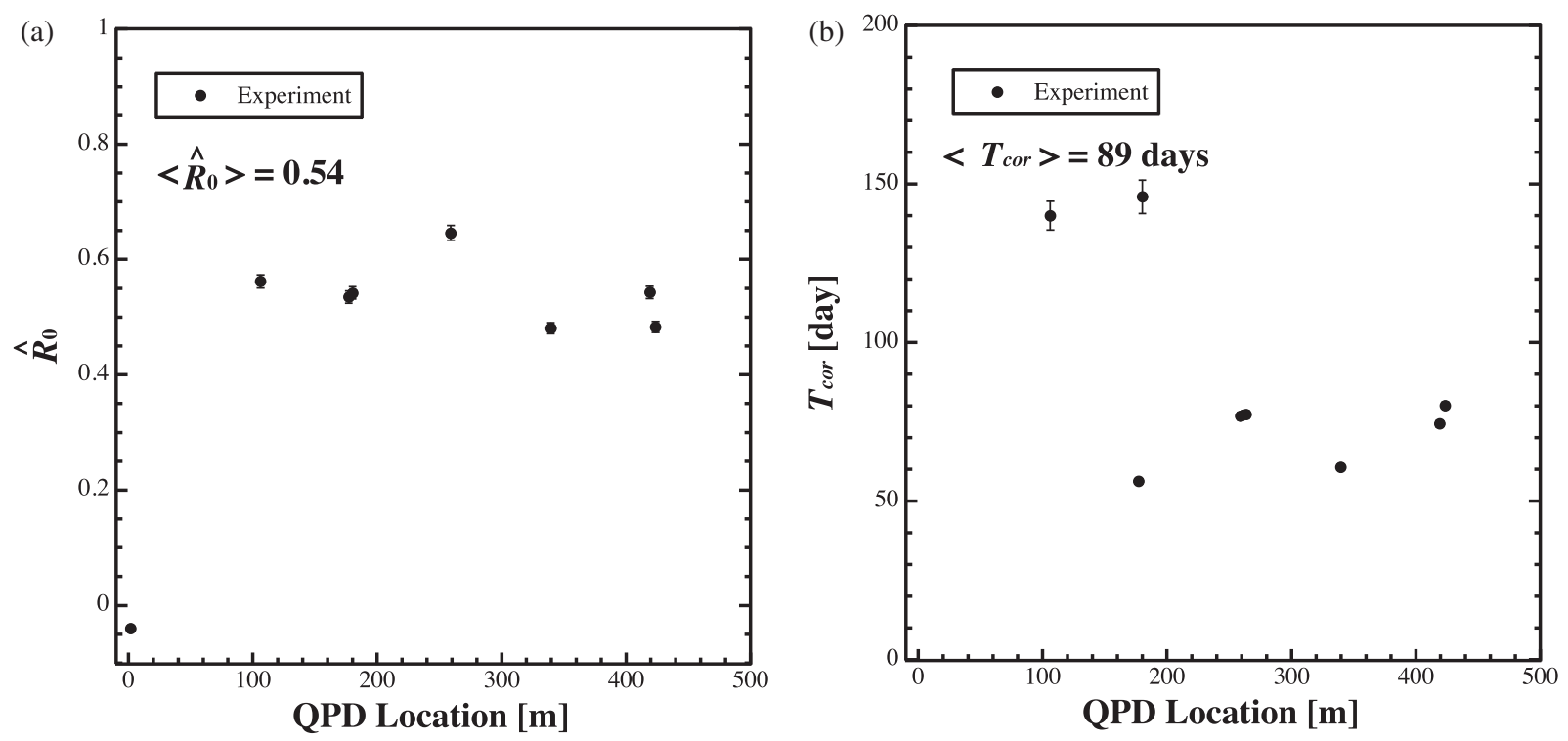

FIG. 15. Variations of (a) $\hat{R}_{0}$ and (b) $T_{\text {cor }}$ as a function of the QPD location.

It can be seen that each cross-correlation function decays smoothly as a function of the time lag in the corresponding time trace, except for QPD_REF1UA. Assuming an exponentially-decaying curve as a fitting function, the correlation time $\left(T_{\text {cor }}\right)$ for each cross-correlation function can be calculated. The average of $T_{\text {cor }}$ is $89 \pm 1$ days, and the results are shown in Fig. 15(b). This means that the temporal cross-correlations also show high coherency in the dynamic displacements of the accelerator units along the entire length of the linac tunnel over relatively long correlation times in the time domain, except for QPD_REF1UA.

Figure 17 shows spatial cross-correlation functions obtained on two different instantaneous dates for all the remote-controllable QPDs. Here, it should be noted that any
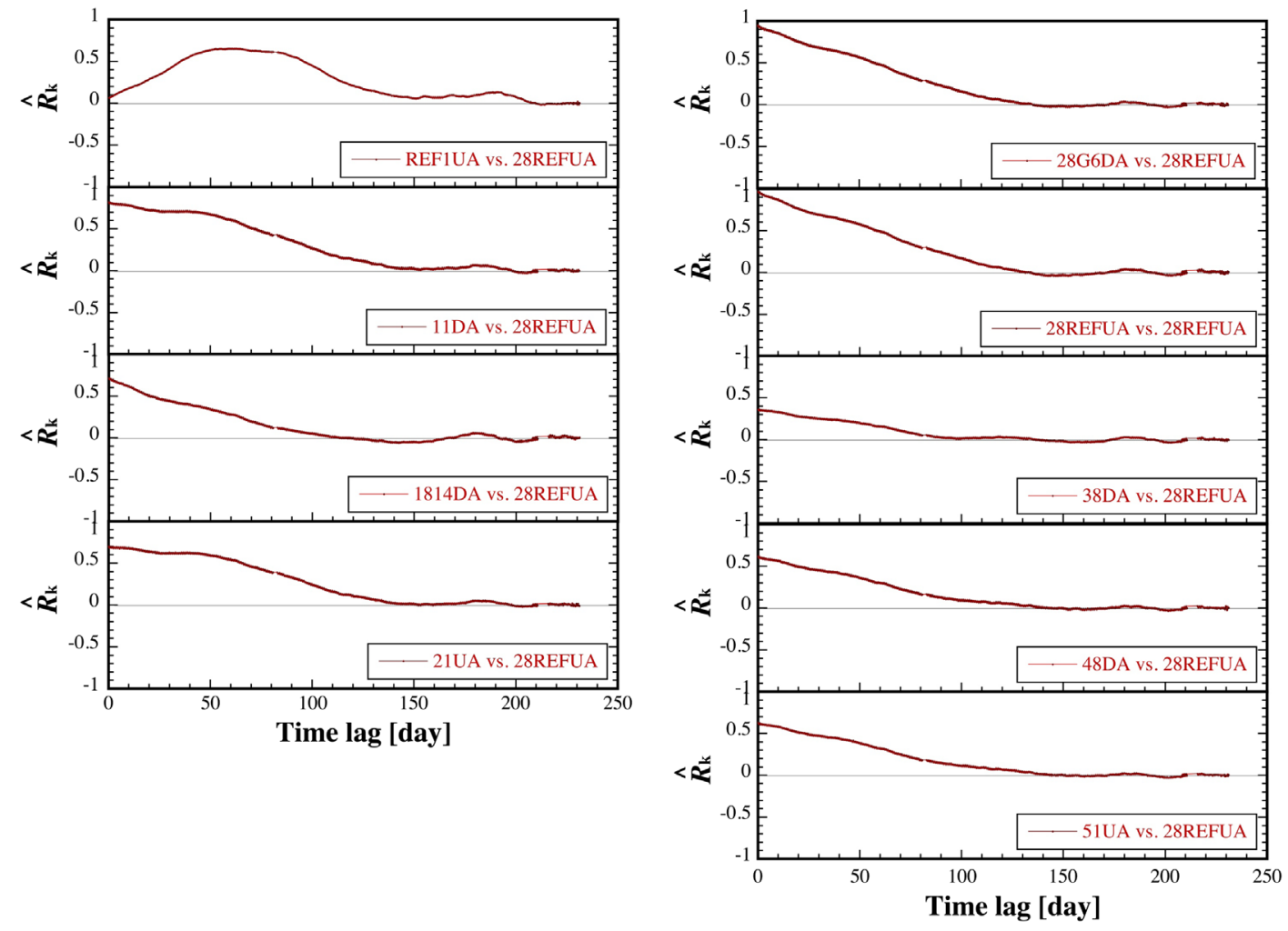

FIG. 16. Time traces in the temporal cross-correlation function of the displacement vectors, for comparisons between the reference QPD and other remote-controllable QPDs for the accelerator units during the same term. 

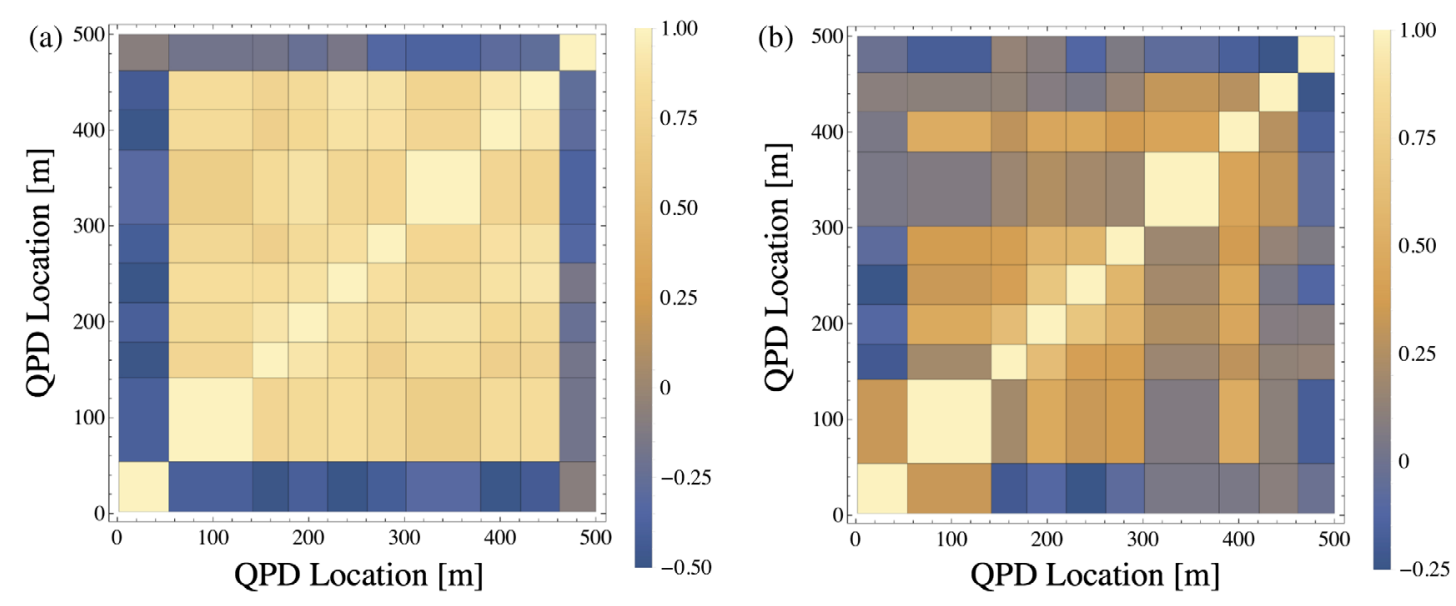

FIG. 17. Intensity distributions in the spatial cross-correlation function between the remote-controllable QPDs in the displacement vector measurements of the accelerator units along the C5 straight section, obtained on (a) 15 Feb 2016 at 0:00 am and (b) 27 Feb 2016 at 8:00 am. The intensity distribution pattern in the cross-correlation function is linearly encoded by the color scale, where bright yellow (dark blue) represents $\hat{R}_{0}$ with the maximum (minimum) value.

correlation function on a straight line tilted at 45 degrees passing through the axis origin is defined as a autocorrelation function, that is, $\hat{R}_{0}=1$. Figure 17 (a) shows that the intensity distribution pattern obtained on 15 Feb 2016 at 0:00 am indicates strong coherency in the dynamic displacement of the floor level. Figure 17(b) shows that a similar distribution pattern obtained on 27 Feb 2016 at 8:00 am indicates less coherency. It should be noted that the cross-correlation functions for QPD_584D $(z=500 \mathrm{~m})$ and QPD_REF1UA $(z=1.74 \mathrm{~m})$ indicate much less coherency, because the laser axis is artificially fixed at the center of QPD_584D with the feedback control on, and because of the isolated behavior of QPD_REF1UA, respectively.

Such complex dynamic displacement phenomena in the spatial and temporal cross-correlation analyses have been markedly understood, and some interesting results were obtained by analyzing the displacement vectors. At the moment, we cannot find any possible origin; however, such dynamic ground motion in the tunnel floor along the entire length of the linac can be observed in real time.

\section{CONCLUSIONS}

A new remote-controllable sensing system for a laserbased alignment system has been developed to measure slow dynamic displacements of the tunnel floor in real time at the KEKB injector linac. The new system was successfully tested with the new detection circuits and control systems. The new calibration scheme for the position sensitivities of the QPDs was also verified, and provided acceptable accuracy.

Using real-time observations obtained through the new system, we observed non-negligible floor displacements caused by dynamic ground motion during an eight-month period. A spatial and temporal cross-correlation analysis of the floor displacements over the entire length of the linac tunnel was performed with the same data. It has become clear that the maximum norms in the displacement vector of the accelerator unit are spread over a range of $0.19-0.88 \mathrm{~mm}$ depending on the QPD location, and the maximum norm is $0.65 \mathrm{~mm}$ on average. The maximum growth rates are spread over a range of 3.3-6.6 $\mu \mathrm{m} /$ day depending on the QPD location, and the maximum growth rate is $4.9 \mu \mathrm{m} /$ day on average.

By analyzing spatial cross-correlations of the displacement vectors, it can be seen that the cross-correlation function varies irregularly and finely over short periods of time; however, the floor levels in each building block move coherently with each other on average over longer periods of time.

By analyzing temporal cross-correlations, it can be seen that the cross-correlation function shows high coherency in the dynamic displacements of the accelerator units along the entire length of the linac tunnel over relatively long correlation times in the time domain. Such real-time observations of the dynamic motion of the tunnel floor could help stabilize the operation of the injector linac.

\section{ACKNOWLEDGMENTS}

The authors would like to thank Professor K. Furukawa of the KEK Accelerator Laboratory for his continuous support of this work. They also thank Mrs. T. Kudou and Y. Mizukawa of Mitsubishi Electric System \& Service Co., Ltd., who helped to conduct this work. This study is fully supported by Ministry of Education, Culture, Sports, Science and Technology (MEXT) of Japan.

[1] V. Parkhomchuk, V. Shiltsev, and G. Stupakov, Slow ground motion and operation of large colliders, Part. Accel. 46, 241 (1994). 
[2] A. Sery and O. Napoly, Influence of ground motion on the time evolution of beams in linear colliders, Phys. Rev. E 53, 5323 (1996).

[3] K. Kubo, Estimation of orbit change and emittance growth due to random misalignment in long linacs, Phys. Rev. ST Accel. Beams 14, 014401 (2011).

[4] T. O. Raubenheimer, Estimates of emittance dilution and stability in high-energy linear accelerators, Phys. Rev. ST Accel. Beams 3, 121002 (2000).

[5] Y. Ohnishi et al., Accelerator design at SuperKEKB, Prog. Theor. Exp. Phys. (2013) 03A011.

[6] T. Abe et al., Achievements of KEKB, Prog. Theor. Exp. Phys. (2013) 03A001.

[7] T. Abe et al., KEK Report No. 2010-1, edited by Z. Doležal and S. Uno.

[8] C. S. Van Heel, in Progress in Optics, edited by E. Wolf (North-Holland, Amsterdam, 1961), Vol. 1, p. 289.

[9] T. Suwada, M. Satoh, and E. Kadokura, Experimental study of new laser-based alignment system at the KEK B-factory injector linear accelerator, Rev. Sci. Instrum. 81, 123301 (2010).

[10] The Stanford Two-Mile Accelerator, edited by R. B. Neal (W. A. Benjamin, New York, 1968), p. 821.

[11] W. B. Herrmannsfeldt, M. J. Lee, J. J. Spranza, and K. R. Trigger, Precision alignment using a system of large rectangular fresnel lenses, Appl. Opt. 7, 995 (1968).

[12] V.E. Bressler, G. E. Fischer, R.E. Ruland, and T. Wang, in Proceedings of the 3rd European Particle Accelerator Conference (EPAC92), Berlin, Germany, 1992, p. 1613, http://www.jacow.org.

[13] C. Schwalm, in 11th International Workshop on Accelerator Alignment (IWAA 2010), Hamburg, 2010, http:// www-conf.slac.stanford.edu/iwaa/pastConf.html.
[14] http://www.xfel.eu.

[15] J. Prenting, in the mini workshop on laser based alignment systems, CERN, Switzerland, 2014, https://indico.cern.ch/ event/285495/timetable/\#20140130.

[16] M. Akemoto et al., The KEKB injector linac, Prog. Theor. Exp. Phys. (2013) 03A002.

[17] I. Abe et al., The KEKB injector linac, Nucl. Instrum. Methods Phys. Res., Sect. A 499, 167 (2003).

[18] M. Kikuchi et al., in Proceedings of the International Particle Accelerator Conference, Kyoto, Japan (ICR, Kyoto, 2010), p. 1641, http://www.jacow.org.

[19] N. Iida, H. Ikeda, T. Kamitani, M. Kikuchi, K. Oide, and D. Zhou, in Proceedings of the 2nd International Particle Accelerator Conference, San Sebastiáán, Spain (EPS-AG, Spain, 2011), p. 2857, http://www.jacow.org.

[20] EPICS homepage, http://www.aps.anl.gov/epics/.

[21] T. Suwada, M. Satoh, S. Telada, and K. Minoshima, Propagation and stability characteristics of a 500-m-long laser-based fiducial line for high-precision alignment of long-distance linear accelerators, Rev. Sci. Instrum. 84, 093302 (2013).

[22] M. Masuzawa, H. Koiso, K. Oide, R. Sugahara, N. Yamamoto, and M. Yoshioka, in Proceedings of the 7th International Workshop on Accelerator Alignment (IWAA 2002) (SPring-8, Japan, 2002), p. 288, http://www-conf .slac.stanford.edu/iwaa/pastConf.html.

[23] V. Shiltsev, Review of observations of ground diffusion in space and in time and fractal model of ground motion, Phys. Rev. ST Accel. Beams 13, 094801 (2010).

[24] G. Kitagawa, Introduction to Time Series Modeling, Monographs on Statistics \& Applied Probability 114 (Chapman \& Hall/CRC Press, New York, 2010), p. 17. 